\title{
Laparoscopy-Assisted Right Hepatectomy and Caudate Lobectomy with Portal Reconstruction for Hilar Cholangiocarcinoma
}

\author{
Osamu Itano, M.D., Ph.D. \\ Department of Surgery, Keio University, School of Medicine, Tokyo, Japan. \\ E-mail: laplivertiger@gmail.com
}

\section{Go Oshima, M.D.}

Department of Surgery, Eiju General Hospital, Tokyo, Japan.

Shingo Maeda, M.D., Ph.D.

Department of Surgery, Eiju General Hospital, Tokyo, Japan.

Naokazu Chiba, M.D., Ph.D.

Department of Surgery, Eiju General Hospital, Tokyo, Japan.

Satoshi Aikou, M.D., Ph.D.

Department of Surgery, Eiju General Hospital, Tokyo, Japan.

Yuko Kitagawa, M.D., Ph.D.

Department of Surgery, Keio University, School of Medicine, Tokyo, Japan.

(c) Mary Ann Liebert, Inc. DOI: 10.1089/vor.2011.0054
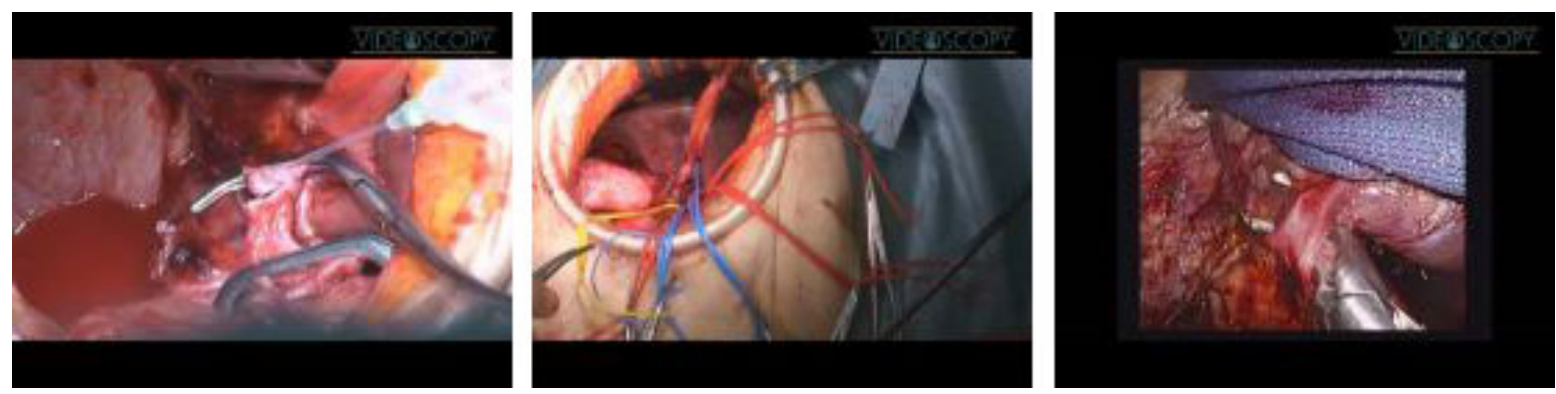

\section{Abstract}

Introduction: Laparoscopic resection of hilar cholangiocarcinoma remains uncommon because of its technical difficulties. ${ }^{1,2}$ However, the main site of the operation for hilar cholangiocarcinoma is the right subcostal area, and most procedures, including major hepatectomy and even vascular reconstruction, can be performed through a $10-\mathrm{cm}$ right subcostal incision if mobilization of the liver is carried out laparoscopically. This video demonstrates our novel minimally invasive technique of laparoscopyassisted right hepatectomy and caudate lobectomy with portal reconstruction for advanced hilar cholangiocarcinoma.

Materials and Methods: The patient in the video was an 82-year-old woman who was diagnosed to have hilar cholangiocarcinoma (Bismuth type IV). The patient was placed in left hemilateral position to switch easily between the supine and left lateral position. First, a 10-cm minilaparotomy incision was made at the right subcostal margin. During lymphadenectomy of the hepatoduodinal ligament, the feasibility of the operation was finally confirmed, including the possibility of vascular reconstruction. In the video case, there was portal vein invasion at the level of the bifurcation. Then the right hepatic artery and the common bile duct were divided. Gelport (Applied Medical, Rancho Santa Margarita, CA) was put on the subcostal incision and laparoscopic mobilization of the liver was performed with four trocars added. In addition to 
the mobilization of the lateral segment and the right lobe, the caudate lobe was completely mobilized from the inferior vena cava. A parenchymal transection was performed using a modified hanging maneuver through the minilaparotomy incision, using the same method as for open liver resection. The left hepatic duct was divided adjacent to the umbilical portion. As we reach the posterior surface of liver, the right hepatic vein was dissected and divided using a linear stapler. After clamping the portal trunk and left portal branch as far from the portal bifurcation as possible, the portal vein was resected and the specimen was retrieved through the minilaparotomy. Portal reconstruction was performed in an end-to-end fashion. Roux-en-Y hepaticojejunostomy was finally performed.

Results and Conclusions: A total of three cases underwent laparoscopy-assisted right hepatectomy and caudate lobectomy (with portal reconstruction in one case). The mean operative time was 672 minutes and the mean blood loss was $1047 \mathrm{~mL}$. There were no intraoperative complications. Histological examination of resected specimens revealed curative status with clear margins in all cases. We conclude that this operation is feasible and safe. It should be currently performed with appropriate expertise in both hepatobiliary and minimally invasive surgery. Larger trials are needed to determine whether this approach offers any advantage over the standard open approach.

The authors have nothing to disclose.

Runtime of video: 7 mins 51 secs

\section{Cite this video}

Osamu Itano, Go Oshima, Shingo Maeda, Naokazu Chiba, Satoshi Aikou, Yuko Kitagawa, LaparoscopyAssisted, Right Hepatectomy and Caudate Lobectomy with Portal Reconstruction for Hilar Cholangiocarcinoma, J Laparoendosc Adv Surg Tech Part B Videoscopy. 2011, DOI: 10.1089/vor.2011.0054.

\section{References}

1. Giulianotti PC, Sbrana F, Bianco FM, Addeo P. Robot-assisted laparoscopic extended right hepatectomy with biliary reconstruction. J Laparoendosc Adv Surg Tech A 2010;20:159-163.

2. Yu H, Wu SD, Chen DX, Zhu G. Laparoscopic resection of Bismuth type I and II hilar cholangiocarcinoma: an audit of 14 cases from two institutions. Dig Surg 2011;28:44-49.

Original Publication Date: 2011 